\title{
Patient-Reported Shoulder Morbidity and Fatigue among Breast Cancer Survivors: An Insight from a Tertiary Care Cancer Hospital
}

\begin{abstract}
Context: Breast cancer is the most common cancer in Indian women with an annual mortality of around 87,000. Treatment for breast carcinoma may lead to swelling of the ipsilateral arm, shoulder stiffness, arm pain, and cancer-related fatigue. Very few centers in India have reported the arm and shoulder morbidity treated in their hospitals. Aims: The aim was to evaluate the predictive factors of arm and shoulder morbidity and fatigue among breast cancer survivors. Settings and Design: This was a retrospective analysis based on a prospectively maintained database. Materials and Methods: Early and locally advanced cases of breast cancer patients were screened for the study during 2015-2018. Eligible participants were invited to fill up the predetermined questionnaire, and their demographic and treatment-related information was accrued from a file archive. Follow-up period was estimated from the date of tissue diagnosis to last contact/time of interview. Results: Shoulder stiffness was the most common complaint followed by arm numbness. Obesity and diabetes played a crucial role in most of the morbidities and fatigue. The median fatigue score was 34 , and the median time of appearance of lymphedema was 13 months. Modified radical mastectomy and radiotherapy to axilla were statistically significantly $(P=0.04$ and 0.01 , respectively) associated with greater shoulder stiffness and arm swelling. Conclusions: Obesity, diabetes, type of surgery, the extent of axillary dissection, and radiation plan are the major predictive factors of arm and shoulder morbidity. Further prospective validation is necessary for future breast cancer survivorship programs.
\end{abstract}

Keywords: Breast cancer, patient-reported outcome, shoulder morbidity, survivorship

\section{Introduction}

Breast cancer is the most frequently diagnosed cancer in women across the globe. India has registered a staggering number of 162,468 new cases with an annual mortality of $12.11 \%$ in $2018 .^{[1]}$ The multimodality treatment approach in the form of surgery, chemotherapy, and radiotherapy revolves around the core management of early to locally advanced breast carcinoma. Although these treatments have improved clinical outcomes, they have been associated with substantial adverse effects. These treatment strategies are associated with many short and long-term complications such as shoulder pain, decreased range of motion, lymphedema, numbness, and fatigue. ${ }^{[2-4]}$ Shoulder morbidities can appear at varied times after surgery and may persist in the long run. ${ }^{[5,6]}$ Lymphedema, a sequela of breast cancer disease as well as its management, changes patient's functional abilities and psychosocial health

\footnotetext{
This is an open access journal, and articles are distributed under the terms of the Creative Commons Attribution-NonCommercial-ShareAlike 4.0 License, which allows others to remix, tweak, and build upon the work non-commercially, as long as appropriate credit is given and the new creations are licensed under the identical terms.
}

For reprints contact: WKHLRPMedknow_reprints@wolterskluwer.com as a constant reminder of the suffering. Breast cancer-related lymphedema occurs as a consequence of various risk factors such as the number of axillary lymph node excised, regional nodal irradiation, obesity, and decreased compliance to physiotherapy and exercise, but a clear consensus on its exact etiology is still lacking and likely to be multifactorial. ${ }^{[7]}$ Although the incidence of lymphedema has decreased from $63 \%$ to $16 \%$ in the last three decades, it continues to be a major complication even with the most advanced treatments. ${ }^{[8,9]}$ Various population-based registries have documented their findings regarding breast cancer-related lymphedema; most of them are retrospective in design with contradictory findings. In general, lymphedema appears within the first 2 years of treatment (surgery and adjuvant radiotherapy) with an average duration of 14 months. $^{[10]}$

Cancer-related fatigue, on the other hand, is frequently experienced by the survivors in the form of weakness, insomnia, and

How to cite this article: Basu A, Mondal J, Swetha B, Chakrabarty S, Ghosh D, Gangopadhyay S, et al. Patient-reported shoulder morbidity and fatigue among breast cancer survivors: An insight from a tertiary care cancer hospital. Indian J Med Paediatr Oncol 2020;41:825-31.

\author{
Abhishek Basu, \\ Janmenjoy Mondal, \\ Bhukya Swetha, \\ Shinjini \\ Chakrabarty, \\ Debjit Ghosh, \\ Subhendu \\ Gangopadhyay, \\ Bidyut Mandal
}

Department of Radiotherapy, Medical College and Hospital, Kolkata, West Bengal, India

Submitted: 24-May-2020

Revised: 05-Sep-2020

Accepted: $11-$ Sep-2020

Published: 31-Dec-2020

Address for correspondence: Dr. Janmenjoy Mondal, Room No. 13, Department of Radiotherapy, Medical College, Kolkata, West Bengal, India. E-mail: janmenjoymondal268@ gmail.com

Access this article online

Website: www.ijmpo.org

DOI: 10.4103/ijmpo.ijmpo_257_20 Quick Response Code:

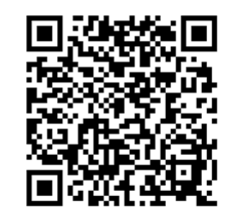


depression, thus posing a major threat to the quality of life. ${ }^{[1]}$ The remarkable survival benefit in the past few years has also thrown a pertinent question regarding the quality of life among breast cancer survivors. Indian data on shoulder morbidity among breast cancer survivors are not very encouraging. This study aimed to elucidate the predictive factors of shoulder morbidity and fatigue and its association with the clinical outcome in a tertiary academic cancer hospital in India.

\section{Materials and Methods}

Breast cancer survivors, attending their routine follow-up visits at radiotherapy outpatient department (OPD) of our hospital, were requested to be included in this study. Participants were selected as per predefined inclusion and exclusion criteria (see below) between the periods of January 2015 and December 2018 and traced till December 2019. Informed consent was obtained in all cases before the collection of data after a thorough explanation of the study. Personal information was de-identified, and each participant was allotted a study number.

\section{Inclusion criteria}

a. Patients with histologically proven carcinoma breast of early and locally advanced stages based on AJCC $8^{\text {th }}$ staging

b. Female patients aged between 18 and 65 years

c. Patients with adequate performance status (Karnofsky performance status $>60$ )

d. Patients with provision of informed consent.

\section{Exclusion criteria}

a. Patients with malignant phyllodes tumor of the breast

b. Known case of rheumatoid arthritis, cervical spondylitis, or those with any previous history of shoulder injury or pathology

c. Those who had undergone prior chemotherapy, radiotherapy, or surgery for this or any other malignancy

d. Those with current or previous history of reconstructed breast surgery.

Participants were asked to complete the predetermined questionnaire in their language. Patient's caregivers aided them if needed, and a nonmedical staff was kept in standby. The questionnaire focused on symptoms of shoulder morbidity such as pain, numbness, and decreased range of motion, in simple terms in three languages (English, Hindi, and Bengali). The questionnaire was inspired by the Australian Breast Cancer Family Study, ${ }^{[11]}$ and fatigue was assessed by interviewing with the FACIT Fatigue Scale (Version 4). ${ }^{[12]}$ Those with symptoms that appeared within the first 6 months from surgery were excluded to avoid confounding and to gauge the long-term burden.

Baseline demographic characteristics and treatment-related data including tumor size, TNM stage (AJCC $8^{\text {th }}$ ), tumor grade, type of surgery, status of axillary clearance, nodal yield (ratio of number of positive nodes to total number of nodes removed), status of neoadjuvant and/or adjuvant chemotherapy, and field, as well as dose fractionation schedules of radiotherapy, were retrospectively collected from a prospectively maintained database. Patients of breast cancer were encouraged to pursue physical exercises and physiotherapy through thorough referral to physical medicine and rehabilitation (PMR) department as a routine institutional protocol, and their compliance to such advices was also noted from file archives. Follow-up period was defined from the date of histopathologic diagnosis to the date of last contact or death. Response assessment was done as per RECIST criteria (version 1.1) based on the data from the file archives.

\section{Statistical considerations and data analysis}

Five hundred and sixty consecutive patients of breast cancer, registered during the study period, were screened, and only 364 patients were invited based on inclusion/ exclusion criteria. Fourteen patients turned down the invitation and eight responses were incomplete, so finally 342 patients' self-reported responses were accrued for final analysis. Demographic and clinical data were analyzed by descriptive statistics, where categorical variables were reported in frequencies and percentages and numerical variables were described by the mean and standard deviation. Predictive factors of shoulder morbidity and fatigue were analyzed by independent sample $t$-test and univariate analysis. Significant predictive factors were selected for further validation in the multivariate Cox regression model. In the Cox regression analysis, hazard ratios and 95\% confidence intervals were calculated to summarize the effect of each factor on fatigue and disability outcomes. Cox regression model is a method for investigating the effect of several variables upon the time a specified event takes to happen. If the hazard ratio is $<1$, the factor is protective; if it is $>1$, then the factor is associated with increased risk or decreased survival. Age, body mass index (BMI), and treatment-related factors (such as type of surgery and radiation field) were adjusted covariates because they were fixed for a patient, not changed over time. Outcome variables such as shoulder stiffness, arm pain/swelling, numbness, and fatigue were time-dependent variables. Participants who did not develop the symptoms under study at the end of their follow-up were censored. Survival analysis was done by the Kaplan-Meier test along with a log-rank test for comparison between groups. $P<0.05$ was considered statistically significant. All the statistical analyses were done using IBM SPSS software version 23 (Chicago, IL, USA, 2015).

\section{Results}

With a median follow-up of 37 months, the median age of the participants was 47 years. Stage II and III cases predominated as per inclusion criteria; other baseline demographic and clinical characteristics were comparable 
in the participants [Table 1]. The median diagnostic delay (appearance of symptoms to tissue diagnosis) was 8 months. We observed that $9.2 \%$ of the patients underwent wide local excision, $33.2 \%$ underwent breast-conserving surgery + axillary clearance, and 57.6\% underwent modified radical mastectomy (MRM) [Table 2]. Adequate axillary dissection ( $>10$ lymph nodes removed) was noted in $53.5 \%$ cases, while $1 \%$ of them were associated with extracapsular extension. The study also revealed that $85.4 \%$ and $81.9 \%$ of patients received chemotherapy and radiotherapy, respectively. The median external beam radiotherapy dose was $50 \mathrm{~Gy}$, and the majority of the cases $(92 \%)$ were treated with two-dimensional radiotherapy by a Telecobalt machine (780C Theratron, Atomic Energy of Canada Ltd (AECL), Ontario, Canada). Only $15.5 \%$ of the patients received tumor boost radiation as per indication. The association of shoulder morbidity and fatigue with the predictive factors is depicted in Tables 2 and 3. We have noticed that $91.35 \%$ of survivors attended PMR OPD for consultation, of which $76.27 \%$ had revisited after initial referral and $27.45 \%$ of the participants required assisted physiotherapy for more arm swelling and arm and shoulder pain.

\section{Shoulder stiffness}

Two hundred and eighteen (63.7\%) participants reported shoulder stiffness anytime during the 6 months after diagnosis till the time of evaluation. Among them, 117 (34.2\%) patients reported stiffness during radiotherapy and 93 (27.2\%) patients reported severe stiffness in any of the period. On physical examination, sixty $(18.4 \%)$ patients had a noticeable decrease in range of motion and still complained of shoulder stiffness even after the best possible exercise on the treated side. We noticed that patients with high BMI $(P=0.01)$, patients with diabetes $(P=0.01)$, and patients who underwent MRM $(P=0.04)$ and axillary radiation $(10 \%$ more) were more prone to develop ipsilateral shoulder stiffness $(P=0.01)$ [Table 2]. There was no statistically significant association with radiotherapy dose fractionation and extent of axillary clearance ( $P=0.20$ and 0.41 , respectively).

\section{Arm swelling}

Assessment of arm swelling/edema was made by careful visual inspection of hands, forearms, and upper arms, with the arms down, abducted $90^{\circ}$, and elevated above the head. This study found that $144(42.1 \%)$ patients reported arm swelling (ipsilateral) anytime during the 6 months after diagnosis till now. The median time of development of lymphedema was 13 months. We noted that $11 \%$ of the sufferers had reported severe disability, while $64.5 \%$ of the patients had very little impact on carrying out the daily activity [Table 4]. MRM $(P=0.03)$, axillary radiation $(P=0.01)$, and a greater number of nodal

Table 1: Participants' demographic and clinical characteristics

\begin{tabular}{|c|c|c|}
\hline Variable & Number of participants & Percentage (median) \\
\hline \multicolumn{3}{|l|}{ Age (years) } \\
\hline$<40$ & 167 & 48.83 (47 years) \\
\hline$>40$ & 175 & 51.17 \\
\hline Residence (urban/semi-urban/rural) & $133 / 143 / 66$ & $38.9 / 41.8 / 19.30$ \\
\hline Education (primary/secondary/HS/graduate) & $171 / 80 / 54 / 37$ & $50 / 23.4 / 15.8 / 10.8$ \\
\hline \multicolumn{3}{|l|}{ Comorbidity } \\
\hline Diabetes mellitus & 87 & 25.4 \\
\hline Hypertension & 129 & 37.7 \\
\hline Missing & 126 & 36.9 \\
\hline Parity: nulliparous/parous & $46 / 296$ & $13.45 / 86.54$ \\
\hline \multicolumn{3}{|l|}{$\operatorname{BMI}\left(\mathrm{kg} / \mathrm{m}^{2}\right)$} \\
\hline$<18.5$ & 47 & $13.74(26.50)$ \\
\hline $18.5-24.9$ & 85 & 24.85 \\
\hline $25-29.9$ & 97 & 28.36 \\
\hline$\geq 30$ & 110 & 32.16 \\
\hline \multicolumn{3}{|l|}{ Affected side } \\
\hline Right & 158 & 46.2 \\
\hline Left & 184 & 53.8 \\
\hline Tumor grade $(1 / 2 / 3)$ & $32 / 198 / 112$ & $9.4 / 57.9 / 32.7$ \\
\hline Stage (I/II/III) & $31 / 115 / 196$ & $9.0 / 33.6 / 57.3$ \\
\hline \multicolumn{3}{|l|}{ Molecular subtype } \\
\hline Luminal A/luminal B/Her2+/TNBC & $124 / 98 / 75 / 45$ & $36.3 / 27.7 / 23.8 / 12.2$ \\
\hline \multicolumn{3}{|l|}{ Nodal status at diagnosis (clinicopathological) } \\
\hline Node positive & 286 & 83.62 \\
\hline Node negative & 50 & 14.61 \\
\hline Missing value & 6 & 1.75 \\
\hline
\end{tabular}

BMI - Body mass index; HS - Higher secondary 


\begin{tabular}{|c|c|c|c|c|}
\hline Variables (percentage of case) $(n=342)$ & Shoulder stiffness count $(P)$ & Arm swelling count $(P)$ & $\begin{array}{c}\text { Arm } \\
\text { numbness } \\
\text { count }(P)\end{array}$ & $\begin{array}{l}\text { Arm pain } \\
\text { count }(P)\end{array}$ \\
\hline \multicolumn{5}{|l|}{ Age group } \\
\hline$<40$ years $(48.8)$ & $112(P=0.3)$ & $68(P=0.7)$ & $94(P=0.4)$ & $68(P=0.6)$ \\
\hline$>40$ years $(51.2)$ & 106 & 76 & 114 & 70 \\
\hline \multicolumn{5}{|l|}{ Time of appearance } \\
\hline 6 months-1 year & 35 & 56 & 26 & 22 \\
\hline $1-2$ years & 82 & 79 & 72 & 60 \\
\hline$>2$ years & 101 & 09 & 110 & 56 \\
\hline$P$ & 0.4 & 0.6 & 0.7 & 0.2 \\
\hline Diabetes mellitus (25.4) & $67(P<0.05)$ & $34(P<0.05)$ & 80 & $28(P<0.05)$ \\
\hline$n=86$ & & & $(P<0.05)$ & \\
\hline \multicolumn{5}{|l|}{ BMI } \\
\hline 18.5- 24.9 & 90 & 69 & 100 & 77 \\
\hline 25 or more & 128 & 75 & 108 & 61 \\
\hline$P$ & 0.04 & 0.06 & 0.7 & 0.4 \\
\hline \multicolumn{5}{|l|}{ Type of surgery } \\
\hline WLE (9.2) & 21 & 12 & 23 & 08 \\
\hline BCS+ALND (33.2) & 89 & 48 & 68 & 63 \\
\hline $\operatorname{MRM}(57.6)$ & 108 & 84 & 117 & 67 \\
\hline$P$ & 0.04 & 0.01 & 0.05 & 0.04 \\
\hline \multicolumn{5}{|l|}{ Radiotherapy fields } \\
\hline $2(\mathrm{CW})(33.6)$ & 78 & 34 & 96 & 64 \\
\hline $3(\mathrm{CW}+\mathrm{SCV}+$ axilla) (48.3) & 140 & 110 & 112 & 74 \\
\hline$P$ & 0.01 & 0.01 & 0.05 & 0.06 \\
\hline \multicolumn{5}{|l|}{ Radiotherapy dose fractionation } \\
\hline Conventional (52.3) & 149 & 74 & 128 & 70 \\
\hline Hypo-fractionation (29.6) & 69 & 70 & 80 & 68 \\
\hline$P$ & 0.2 & 0.06 & 0.08 & 0.06 \\
\hline \multicolumn{5}{|l|}{ Chemotherapy } \\
\hline Neoadjuvant (36.4) & 101 & 66 & 86 & 77 \\
\hline Adjuvant* (72.2) & 117 & 78 & 122 & 61 \\
\hline Missing value (14.6) $(P)$ & 0.6 & 0.3 & 0.3 & 0.4 \\
\hline \multicolumn{5}{|l|}{ Additional adjuvant therapy } \\
\hline Hormone (64) & 166 & 83 & 148 & 84 \\
\hline Trastuzumab (20.1) & 52 & 61 & 60 & 54 \\
\hline$P$ & 0.03 & 0.06 & 0.07 & 0.08 \\
\hline
\end{tabular}

*Adjuvant cases also include some cases who received neoadjuvant chemotherapy also. CW - Chest wall; SCV - Supraclavicular; WLE - Wide local excision; BCS - Breast-conserving surgery; ALND - Axillary nodal dissection; MRM - Modified radical mastectomy. Univariate analysis test was used; BMI - Body mass index

dissection $(P=0.01)$ were associated with significant lymphedema [Tables 2 and 3].

\section{Numbness of arm}

Two hundred and eight $(60.8 \%)$ patients reported numbness anytime during the 6 months after diagnosis till now. Among them, $126(60.57 \%)$ patients reported numbness during radiotherapy and $82 \quad(39.42 \%)$ patients still complained of numbness. Only $15.5 \%$ of the sufferers had complained about significant disability [Table 4]. Diabetes mellitus $(P=0.02)$ and type of surgery $(0.01)$ were noted as predictive factors of arm numbness.

\section{Pain/ache in the arm}

One hundred and thirty-eight participants had reported pain in the arm and $27.7 \%$ of them complained moderate-to-severe pain. The majority of them have developed the symptoms at least a year after the diagnosis. Diabetes mellitus and type of surgery had registered increasing risk for developing the morbidity $(P<0.05)$. Hypo-fractionated radiotherapy was associated with a $36 \%$ lower risk of developing pain in the arm but was not statistically significant $(P=0.06)$ [Tables 2 and 3]. Multivariate analysis turned down such correlations barring diabetes mellitus. 


\section{Fatigue}

Fatigue was assessed by FACIT Fatigue Scale (Version 4). It was assessed on an one-to-one interview basis. The scores lie between 0 and 52 theoretically. The median fatigue score is 34 (14-42 in our study). Independent sample $t$-test revealed that fatigue in survivors correlated with high BMI, diabetes, and multiple lines of adjuvant therapy. On multivariate Cox regression analysis, there was a trend of high fatigue score with high BMI, diabetes, and type of surgery, but none was statistically significant [Table 3].

Tumor stage, tumor grade, molecular subtype, diagnostic delay, age, and laterality (right/left) were associated neither with shoulder morbidity nor fatigue in our study population.

Kaplan-Meier survival analysis had shown that the study population had a 3-year disease-free survival (DFS) of 78\%. The median DFS has not achieved yet; Stage III cases had the lowest DFS of $72.4 \%$ as compared to Stage II $(83.1 \%)$ and Stage I (100\%) [Figure 1].

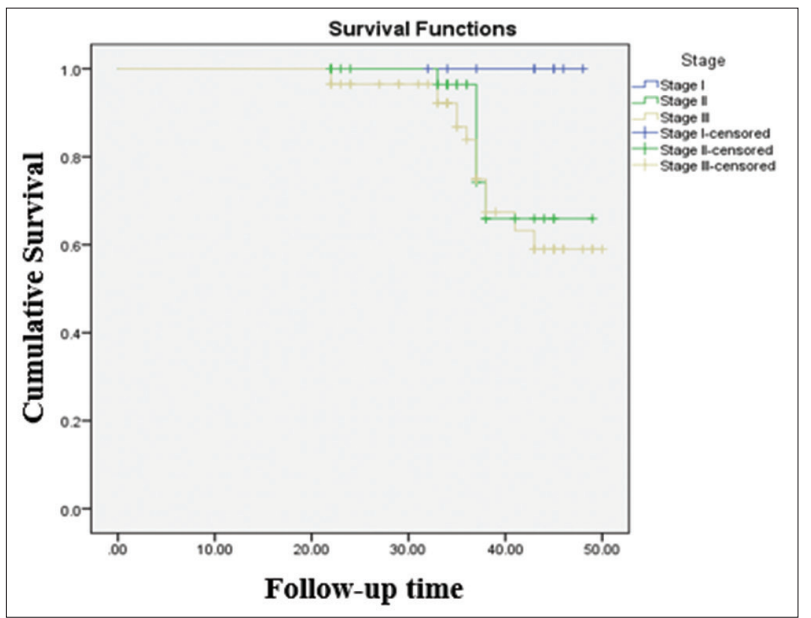

Figure 1: Disease-free survival (stage wise) of the survivors. Log rank value, $P=0.14$

\section{Discussion}

This study reports on breast cancer survivors in a state-sponsored tertiary cancer hospital in eastern India. We observed that shoulder morbidity was the most commonly reported complaint followed by arm numbness and arm swelling and pain. We noted that obesity and diabetes mellitus were significantly associated with shoulder morbidity, arm pain, numbness, and fatigue. This observation was well supported by the published literature, ${ }^{[1,13]}$ although it was not clear whether obesity per se or its associated comorbidities (diabetes and hypertension) reflected such association. This study found that lymphedema (a surrogate for arm swelling) was slightly on the higher side than the previously reported European studies. ${ }^{[14,15]}$ This discrepancy can be explained in terms of racial heterogeneity, compliance to physical exercise, and level of documentation in the patient records. Moreover, the degree of disability and time of appearance varied among the survivors. Type of surgery, the extent of nodal dissection, and axillary radiation were significant covariates of arm swelling in our study. It should be kept in mind that we also incorporated the data from the patients' file with self-reported outcomes and thus avoided the inconsistent reports regarding the predictive factors of lymphedema. ${ }^{[11,13,16]}$ It is noteworthy that a patient cannot understand the field of radiotherapy or the type of surgery, hence overreliance on self-reported outcomes could lead us to a confusing conclusion.

Age was not associated with shoulder and arm morbidity though there is some evidence that older age is more prone to reduced shoulder movement. ${ }^{[17,18]}$ Arm numbness was the second most commonly reported outcome $(60.5 \%)$, and the majority of the survivors have experienced it during or after radiotherapy. Pain in the arm also followed a similar trend. Compromise of the

\begin{tabular}{|c|c|c|c|c|c|}
\hline \multirow{3}{*}{ Variables } & \multicolumn{5}{|c|}{ Table 3: Cox regression analysis of the covariates } \\
\hline & \multicolumn{5}{|c|}{ HR (95\% CI) } \\
\hline & Shoulder stiffness & Arm swelling & Arm numbness & Pain in the arm & Fatigue \\
\hline$\overline{\mathrm{BMI}}$ & $1.42(1.6-2.4)$ & $0.81(0.5-2.2)$ & $0.88(0.5-2.1)$ & $0.92(0.4-1.6)$ & $1.52(0.4-2.1)$ \\
\hline Diabetes mellitus & $1.14(1.2-2.7)$ & $0.91(0.5-2.6)$ & $1.70(1.3-3.2)$ & $1.19(1.3-2.5)$ & $1.81(0.9-2.2)$ \\
\hline Type of surgery & $1.8(1.1-3.4)$ & $1.31(1.8-3.3)$ & $1.24(1.6-3.7)$ & $0.78(0.6-1.5)$ & $1.21(0.4-1.7)$ \\
\hline Radiotherapy dose fractionation & $0.91(0.4-1.8)$ & $1.0(0.4-2.1)$ & $0.81(0.4-2.1)$ & $0.64(0.5-1.1)$ & $1.02(0.4-1.3)$ \\
\hline Field of radiotherapy & $1.1(1.5-2.6)$ & $1.27(1.3-2.1)$ & $0.64(0.4-1.9)$ & $1.17(0.6-1.8)$ & $0.97(0.6-1.5)$ \\
\hline Additional adjuvant therapy & $0.97(0.4-2.1)$ & $0.91(0.5-1.9)$ & $0.89(0.6-1.3)$ & $0.79(0.5-1.1)$ & $0.92(0.6-1.1)$ \\
\hline$>10$ ALND & $1.21(0.4-3.3)$ & $1.95(1.2-3.8)$ & $1.21(0.5-1.9)$ & $1.25(0.6-1.8)$ & $0.99(0.5-1.3)$ \\
\hline \multicolumn{6}{|c|}{ HR - Hazard ratio; ALND - Axillary nodal dissection; CI - Confidence interval } \\
\hline \multicolumn{6}{|c|}{ Table 4: Prevalence of severity of symptoms (\%) among the participants } \\
\hline & \multicolumn{2}{|c|}{ Not at all/very little } & \multicolumn{2}{|c|}{ Quite a lot } & A great deal \\
\hline Shoulder stiffness $(n=218)$ & \multicolumn{2}{|c|}{72.8} & \multicolumn{2}{|c|}{20} & 7.2 \\
\hline Arm swelling $(n=144)$ & \multicolumn{2}{|c|}{64.5} & \multicolumn{2}{|c|}{24.5} & 11 \\
\hline Arm numbness $(n=208)$ & \multicolumn{2}{|c|}{84.5} & \multicolumn{2}{|c|}{10.8} & 4.7 \\
\hline Arm pain $(n=138)$ & \multicolumn{2}{|c|}{72.3} & \multicolumn{2}{|c|}{21.3} & 6.4 \\
\hline
\end{tabular}


intercostobrachial nerve during axillary dissection and nociceptive role of radiotherapy could explain the trend, in accordance with the evidences, ${ }^{[19,20]}$ although whether radiotherapy could cause neurogenic pain is debatable and still under considerable doubt. Consistent with the previous reports, pain in the arm was correlated with axillary nodal dissection, a greater number of lymph nodes dissected, and radiotherapy to the axilla. ${ }^{[21,22]}$ However, it is important to note that Kudel et al., 2007, ${ }^{[23]}$ and Miaskowski et al., 2012, ${ }^{[24]}$ have suggested that persistent pain in the arm or shoulder is associated with preoperative breast pain. We did not explore this point at the time of evaluation; hence, we could not share our thoughts on it. There have been data to suggest that lesser education and lower economic background considerably impact chronic pain. ${ }^{[25,26]}$ Our data could not establish such association and were in line with the evidence suggested by Kudel et al., 2007, and Poleshuck et al., 2006. ${ }^{[27]}$ State-sponsored medication (free of cost) and other economic packages could explain such results in our study cohort.

Laterality (side affected) was not a significant factor of pain in our study unlike others, ${ }^{[28]}$ where it has been hypothesized that the right hemisphere is dominant in processing emotional experience, suggesting a possible link between the right hemisphere and the emotional component of pain processing.

The impact of physical exercise on pain management could not be analyzed as a majority of the participants did not document their response regarding it. However, from the data (physician's note in file archive) in hand, we conclude that the participation and compliance to physical exercise is satisfactory. A prospective evaluation could have avoided this scenario. It is also noteworthy that we did not have a conclusive idea on pain management of the survivors and their association with peripherally inserted central catheter or central venous port.

The majority of the patients had received taxane-based chemotherapy, and it was noted that subsequent lines of chemotherapy (in cases of recurrences) aggravated arm pain. Multiple intravenous cannulation, repeated biopsy, and anxiety could explain this, but the association was not statistically significant $(P=0.06)$, hence was not included in the primary analysis. We admit that a better study design could nullify such errors, and we will keep this in mind. Along with obesity and diabetes, shoulder stiffness, pain in arm, and type of surgery were significantly associated with fatigue.

Several study limitations need to be acknowledged. Although the sample size was representative of the Indian scenario, the retrospective design was a noted caveat. Participants had to recall their symptoms, so a possibility of bias could not be negated. Assessment of preoperative breast pain, number of breast biopsies, compliance to physiotherapy, and physical exercise could not be explored in detail even with our best efforts. We did not measure arm circumference and had to rely upon the patient's perception of arm swelling. Having said that, we believe that our data is robust and possibly the only one which reports shoulder morbidity along with fatigue in breast cancer survivors from India in recent times. This observation is important as it gauges the nuances of the treatment approach and its impact on quality of life among women of different socioeconomic backgrounds.

\section{Conclusions}

We conclude that obesity, diabetes, type of surgery, the extent of axillary dissection, and radiation plan are the major predictive factors of arm and shoulder morbidity. Further prospective validation is necessary for future breast cancer survivorship programs.

\section{Acknowledgment}

We acknowledge the utmost co-operation from our patients and hard work of the physicist team and fellow colleagues.

\section{Financial support and sponsorship}

Nil.

\section{Conflicts of interest}

There are no conflicts of interest.

\section{References}

1. Bray F, Ferlay J, Soerjomataram I, Siegel RL, Torre LA, Jemal A. Global cancer statistics 2018: GLOBOCAN estimates of incidence and mortality worldwide for 36 cancers in 185 countries. CA Cancer J Clin 2018;68:394-424.

2. Freitas-Silva R, Conde DM, de Freitas-Júnior R, Martinez EZ. Comparison of quality of life, satisfaction with surgery and shoulder-arm morbidity in breast cancer survivors submitted to breast-conserving therapy or mastectomy followed by immediate breast reconstruction. Clinics (Sao Paulo) 2010;65:781-7.

3. de Groef A, van Kampen M, Tieto E, Schönweger P, Christiaens MR, Neven $\mathrm{P}$, et al. Arm lymphoedema and upper limb impairments in sentinel node-negative breast cancer patients: A one year follow-up study. Breast 2016;29:102-8.

4. Koehler LA, Blaes AH, Haddad TC, Hunter DW, Hirsch AT, Ludewig PM. Movement, function, pain, and postoperative edema in axillary web syndrome. Phys Ther 2015;95:1345-53.

5. Fleissig A, Fallowfield LJ, Langridge CI, Johnson L, Newcombe RG, Dixon JM, et al. Post-operative arm morbidity and quality of life. Results of the ALMANAC randomised trial comparing sentinel node biopsy with standard axillary treatment in the management of patients with early breast cancer. Breast Cancer Res Treat 2006;95:279-93.

6. Shamley D, Srinaganathan R, Oskrochi R, LascurainAguirrebeña I, Sugden E. Three-dimensional scapulothoracic motion following treatment for breast cancer. Breast Cancer Res Treat 2009;118:315-22.

7. Harris SR, Hugi MR, Olivotto IA, Levine M; Steering Committee for Clinical Practice Guidelines for the Care and Treatment of Breast Cancer. Clinical practice guidelines for the 
care and treatment of breast cancer: 11. Lymphedema. CMAJ 2001;164:191-9.

8. Ferrandez JC, Serin D, Bouges S. Frequency of lymphedema of the upper limb after treatment of breast cancer. Risk factors. Apropos of 683 cases. Bull Cancer 1996;83:989-95.

9. Werner RS, McCormick B, Petrek J, Cox L, Cirrincione C, Gray JR, et al. Arm edema in conservatively managed breast cancer: Obesity is a major predictive factor. Radiology 1991;180:177-84.

10. Suneson BL, Lindholm C, Hamrin E. Clinical incidence of lymphoedema in breast cancer patients in JoÈ nkoÈ ping County, Sweden. Eur J Cancer Care (Engl Lang Ed) 1996;5:7-2.

11. McCredie MR, Dite GS, Porter L, Maskiell J, Giles GG, Phillips KA, et al. Prevalence of self-reported arm morbidity following treatment for breast cancer in the Australian Breast Cancer Family Study. Breast 2001;10:515-22.

12. Acaster S, Dickerhoof R, DeBusk K, Bernard K, Strauss W, Allen LF. Qualitative and quantitative validation of the FACIT-fatigue scale in iron deficiency anemia. Health Qual Life Outcomes 2015;13:60.

13. Edwards TL. Prevalence and aetiology of lymphoedema after breast cancer treatment in Southern Tasmania. Aust N Z J Surg 2000;70:412-8.

14. Mortimer PS, Bates DP, Brassington HD, Stanton AW, Strachan DP, Levick JR. The prevalence of arm oedema following treatment for breast cancer. Q J M 1996;89:377-80.

15. Paci E, Cariddi A, Barchielli A, Bianchi S, Cardona G, Distante $\mathrm{V}$, et al. Long-term sequelae of breast cancer surgery. Tumori 1996;82:321-4.

16. Kissin MW, Querci della Rovere G, Easton D, Westbury G. Risk of lymphoedema following the treatment of breast cancer. $\mathrm{Br} \mathrm{J}$ Surg 1986;73:580-4.

17. Kootstra JJ, Dijkstra PU, Rietman H, de Vries J, Baas P, Geertzen JH, et al. 2013. A longitudinal study of shoulder and arm morbidity in breast cancer survivors 7 years after sentinel lymph node biopsy or axillary lymph node dissection. Breast
Cancer Res Treat 2013;139:125-4.

18. Kramer N, Ramjith J, Shamley D. Prevalence of shoulder morbidity after treatment for breast Cancer in South Africa. Support Care Cancer 2019;27:2591-8.

19. Ivens D, Hoe AL, Podd TJ, Hamilton CR, Taylor I, Royle GT. Assessment of morbidity from complete axillary dissection. Br J Cancer 1992;66:136-8.

20. Andersen KG, Kehlet H. Persistent pain after breast cancer treatment: A critical review of risk factors and strategies for prevention. J Pain 2011;12:725-46.

21. Alves Nogueira Fabro E, Bergmann A, do Amaral E Silva B, Padula Ribeiro AC, de Souza Abrahão K, da Costa Leite Ferreira MG, et al. Post-mastectomy pain syndrome: Incidence and risks. Breast 2012;21:321-5.

22. Caffo O, Amichetti M, Ferro A, Lucenti A, Valduga F, Galligioni E. Pain and quality of life after surgery for breast cancer. Breast Cancer Res Treat 2003;80:39-48.

23. Kudel I, Edwards RR, Kozachik S, Block BM, Agarwal S, Heinberg LJ, et al. Predictors and consequences of multiple persistent postmastectomy pains. J Pain Symptom Manage 2007;34:619-27.

24. Miaskowski C, Cooper B, Paul SM, West C, Langford D, Levine JD, et al. Identification of patient subgroups and risk factors for persistent breast pain following breast cancer surgery. J Pain 2012;13:1172-87.

25. Krueger AB, Stone AA. Assessment of pain: A community-based diary survey in the USA. Lancet 2008;371:1519-25.

26. Cimmino MA, Ferrone C, Cutolo M. Epidemiology of chronic musculoskeletal pain. Best Pract Res Clin Rheumatol 2011;25:173-83.

27. Poleshuck EL, Katz J, Andrus CH, Hogan LA, Jung BF, Kulick DI, et al. Risk factors for chronic pain following breast cancer surgery: A prospective study. J Pain 2006;7:626-34.

28. Shamley D, Lascurain-aguirrebeña I, Oskrochi R. Shoulder morbidity after treatment for breast cancer is bilateral and greater after mastectomy. Acta Oncol (Madr) 2012;51:1045-3. 